\title{
Risk aggregation in non-life insurance : Standard models vs. internal models
}

Kwangmin Jung (Co-authored with Dr. Martin Eling) University of St. Gallen, Switzerland Institute of Insurance Economics

American Risk and Insurance Association (ARIA), 2018 Annual Meeting August $7^{\text {th }}, 2018$, Chicago 
Motivation / Objective and contribution

$\square$ Literature review

$\square$ Methodology

$\square$ Data description

Results \& Applications

\section{$\square$ Conclusion}




\section{Motivation}

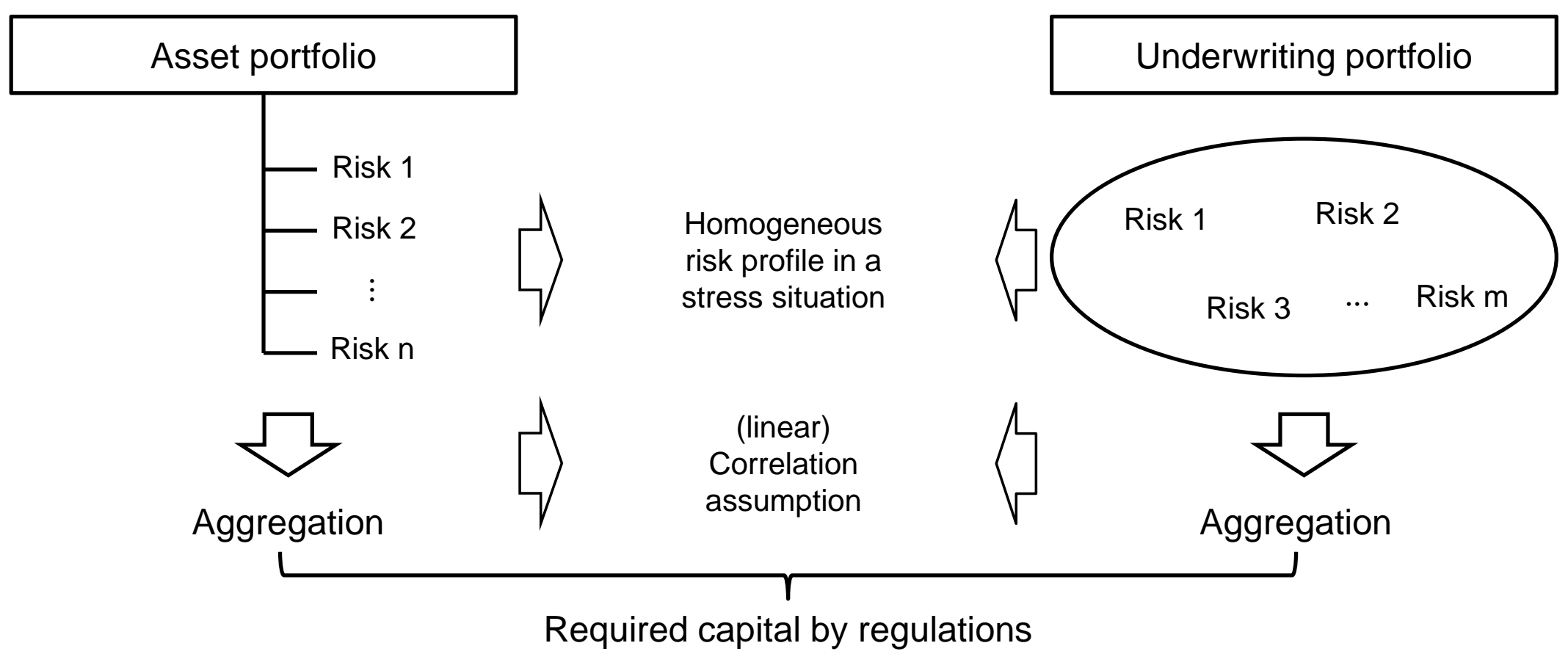

- Thus, there is a need to

(1) construct an undertaking-specific model (internal model) with an efficient, comprehensive modeling

(2) investigate how the impact of considering undertaking-specific risk profile and dependence structures of risk factors is on the risk measurement. 


\section{Objective / Contribution}

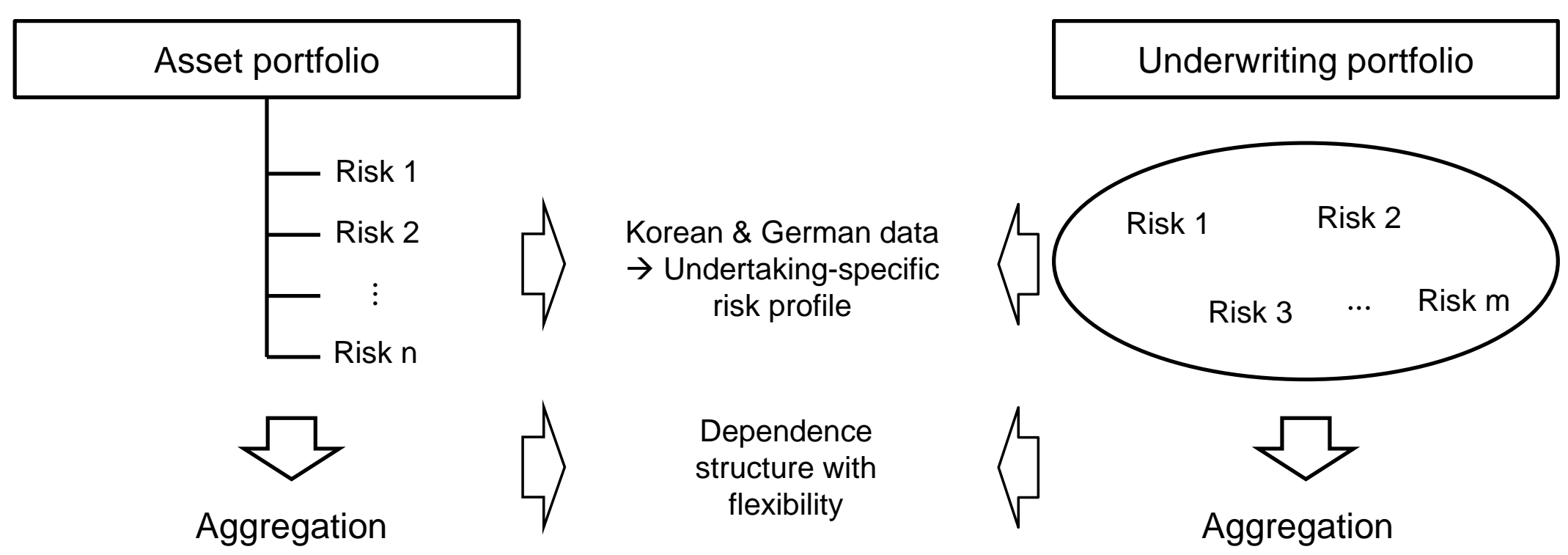

Comparison study with three regulatory frameworks:

Korean Risk-based Capital (RBC), Solvency II and Swiss Solvency Test (SST) 


\section{Overview of the integrated structure}

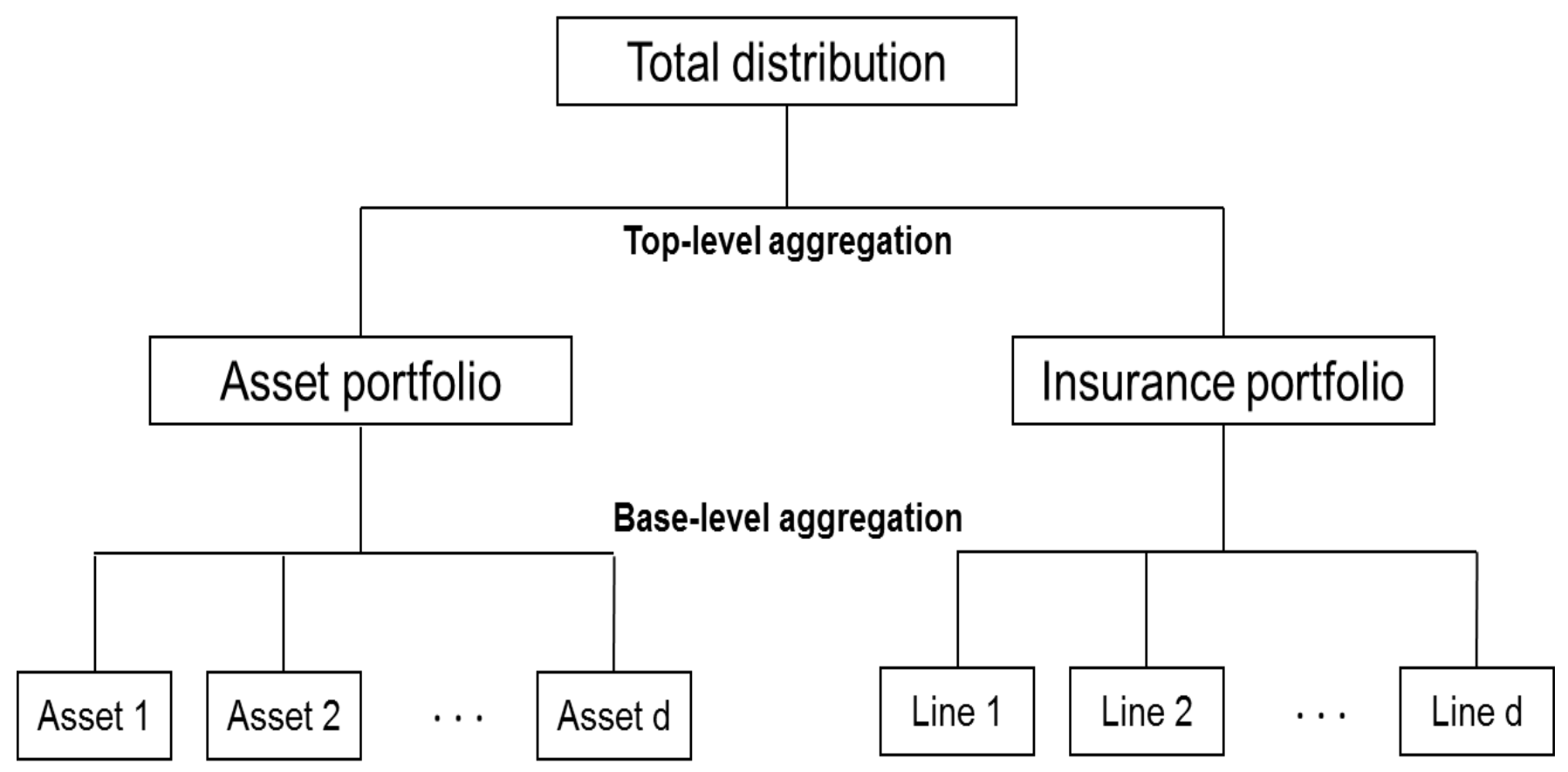




\section{Graphical description on the methodology}

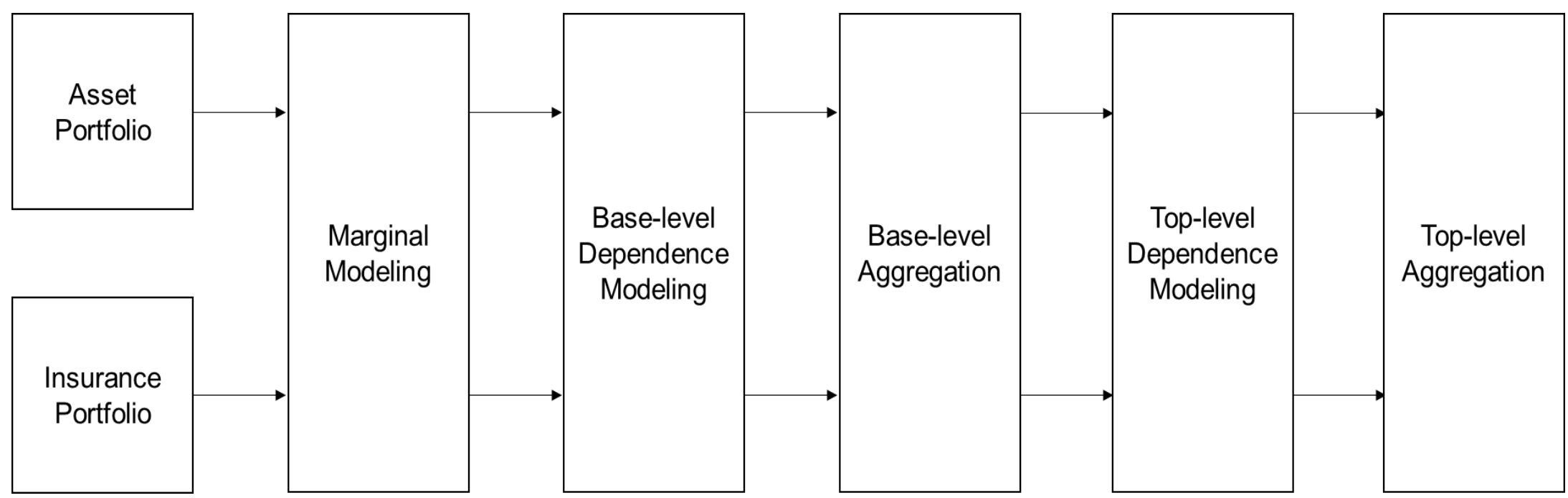




\section{Marginal modeling}

- Asset data: ARMA-GARCH(1,1) with normal, student-t, skew-normal and skew-student

- Underwriting data: Risk modeling (distribution fitting) by testing student-t, skew-normal, skew-student, lognormal, gamma, Weibull, inverse Gaussian, Cauchy, Burr, GPD, POT with normal body and POT with lognormal body.

\section{Dependence modeling}

- Base-level (High-dimension): Elliptical copulas (Gaussian, Student-t), Simple Archimedean (Gumbel, Clayton), Hierarchical (nested) Archimedean (Gumbel, Clayton), Vine copula (R-Vine), Bernstein (D-Vine) and Independence.

- Top-level (Bivariate): Elliptical copulas (Gaussian, Student-t), Simple Archimedean (Gumbel, Clayton) and Independence. 


\section{Dependence modeling}

- List of dependence models used in the empirical study

\begin{tabular}{|c|c|c|c|c|c|}
\hline Model & \# of parameters & Pros & & Cons & \\
\hline Gaussian & $\frac{d(d-1)}{2}$ & $\cdot$ & $\begin{array}{l}\text { Easy to use and interpret. } \\
\text { Normally distributed margins. }\end{array}$ & $\begin{array}{l}\cdot \cdot \\
\cdot\end{array}$ & $\begin{array}{l}\text { No tail dependence. } \\
\text { Limited to symmetric and linear } \\
\text { dependency. }\end{array}$ \\
\hline Student-t & $\frac{d(d-1)}{2}+1$ & $\dot{r}$ & $\begin{array}{l}\text { Easy to use and interpret. } \\
\text { Tail dependence. }\end{array}$ & $\cdot$ & Limited to symmetric dependency. \\
\hline Archimedean & 1 & $\cdot$ & $\begin{array}{l}\text { Easy to construct. } \\
\text { A great variety of copula families } \\
\text { representing diverse dependence } \\
\text { structures exist. }\end{array}$ & & $\begin{array}{l}\text { Difficult interpretation with a single } \\
\text { parameter. } \\
\text { Exchangeability. }\end{array}$ \\
\hline $\begin{array}{l}\text { Hierarchical } \\
\text { Archimedean Copulas } \\
\text { (HAC) }\end{array}$ & $d-1$ & $\cdot$ & $\begin{array}{l}\text { Easy to construct by using generating } \\
\text { functions of Archimedean copulas. } \\
\text { A more accurate dependence structure } \\
\text { by grouping more strongly correlated } \\
\text { variables. }\end{array}$ & $\begin{array}{l}\cdot \\
\cdot \\
\cdot\end{array}$ & $\begin{array}{l}\text { Limited to Archimedean family. } \\
\text { Difficult interpretation. } \\
\text { Complicated form when different } \\
\text { functions are used in a structure. }\end{array}$ \\
\hline $\begin{array}{l}\text { Pair Copula } \\
\text { Construction (PCC) }\end{array}$ & $\frac{d(d-1)}{2}$ & . & $\begin{array}{l}\text { A more accurate high dimensional } \\
\text { dependency by pair-wise dependence } \\
\text { modeling. } \\
\text { A variety of copulas. } \\
\text { Any type of dependence model (either } \\
\text { hierarchical or flexible structure). }\end{array}$ & $\cdot$ & $\begin{array}{l}\text { Difficult interpretation. } \\
\text { Distribution function is not } \\
\text { explicitly available. }\end{array}$ \\
\hline $\begin{array}{l}\text { Bernstein copula } \\
\text { (D-Vine) }\end{array}$ & $\begin{array}{l}\quad \frac{d(d-1)}{2} \cdot(m+1)^{2} \\
\text { where } m \text { is the degree of } \\
\text { Bernstein polynomial }\end{array}$ & $\cdot$ & $\begin{array}{l}\text { Advantageous when parametric copula } \\
\text { functions are misspecified. } \\
\text { Flexible by controlling the polynomial } \\
\text { degree. }\end{array}$ & $\cdot$ & $\begin{array}{l}\text { Still unknown parameter of optimal } \\
\text { polynomial degree } m . \\
\text { Not parsimonious (i.e. }(m+1)^{2} \\
\text { parameters for each bivariate } \\
\text { case in D-Vine). }\end{array}$ \\
\hline
\end{tabular}




\section{Dependence modeling (Base-level) - Graphical structures}

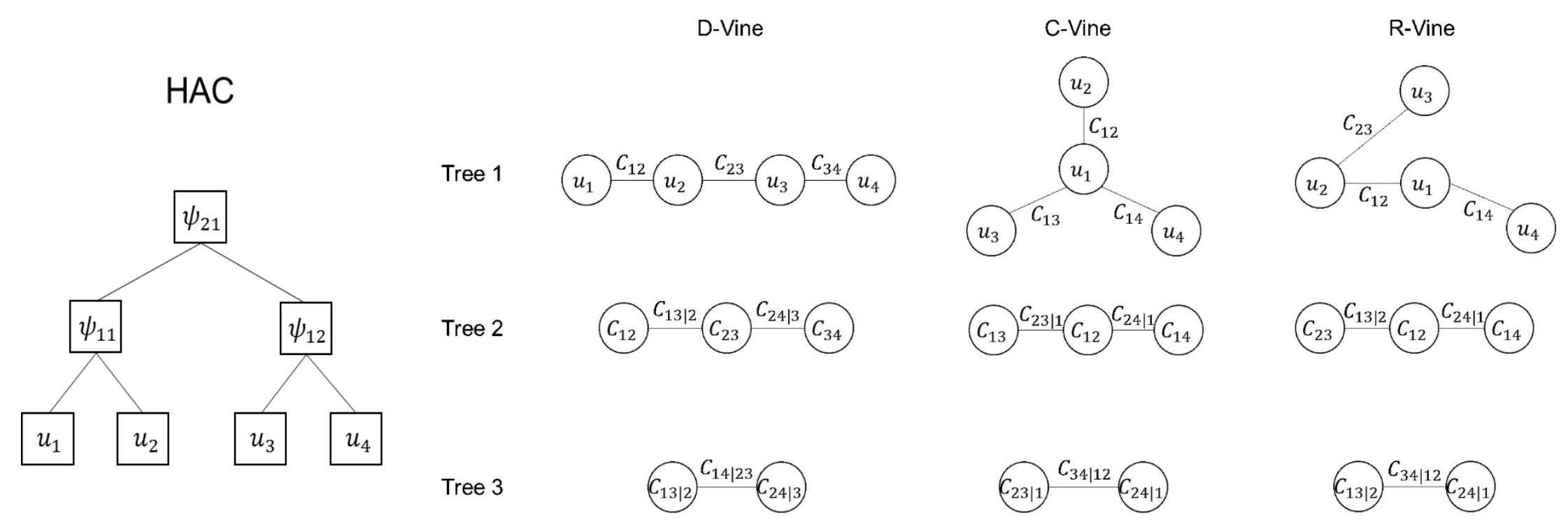




\section{Regulatory frameworks (Korean Risk-Based Capital)}

$$
\text { Required capital }=\sqrt{\sum_{i=1} \sum_{j=1} \operatorname{Corr}_{i, j} \times \operatorname{Risk}_{i} \times \operatorname{Risk}_{j}}
$$

- Factor-based approach

- Risks are estimated by Value-at-Risk $99 \%$ with risk coefficients and calibrated parameters for risk thresholds.

- Correlation matrices are given to between risk modules and between risk factors only within the underwriting module (No assumption on the correlation in the market risk module).

- Market risk capital is calculated by the simple summation of individual risk levels. 


\section{Regulatory frameworks (Solvency II)}

$$
\text { Required capital }=\sqrt{\sum_{i=1} \sum_{j=1} \operatorname{Corr}_{i, j} \times S C R_{i} \times S C R_{j}},
$$

- Factor-based approach

- $\quad$ SCRs are estimated by Value-at-Risk 99.5\% with calibrated parameters for risk thresholds.

- Correlation matrices are given to between risk modules and between risk factors within each risk module.

- The correlation matrix for the market risk module is differentiated by the interest rate shock (up and down turns) 


\section{Regulatory frameworks (Swiss Solvency Test)}

- Model-based approach

- Risks are estimated by tail Value-at-Risk (TVaR) 99\%

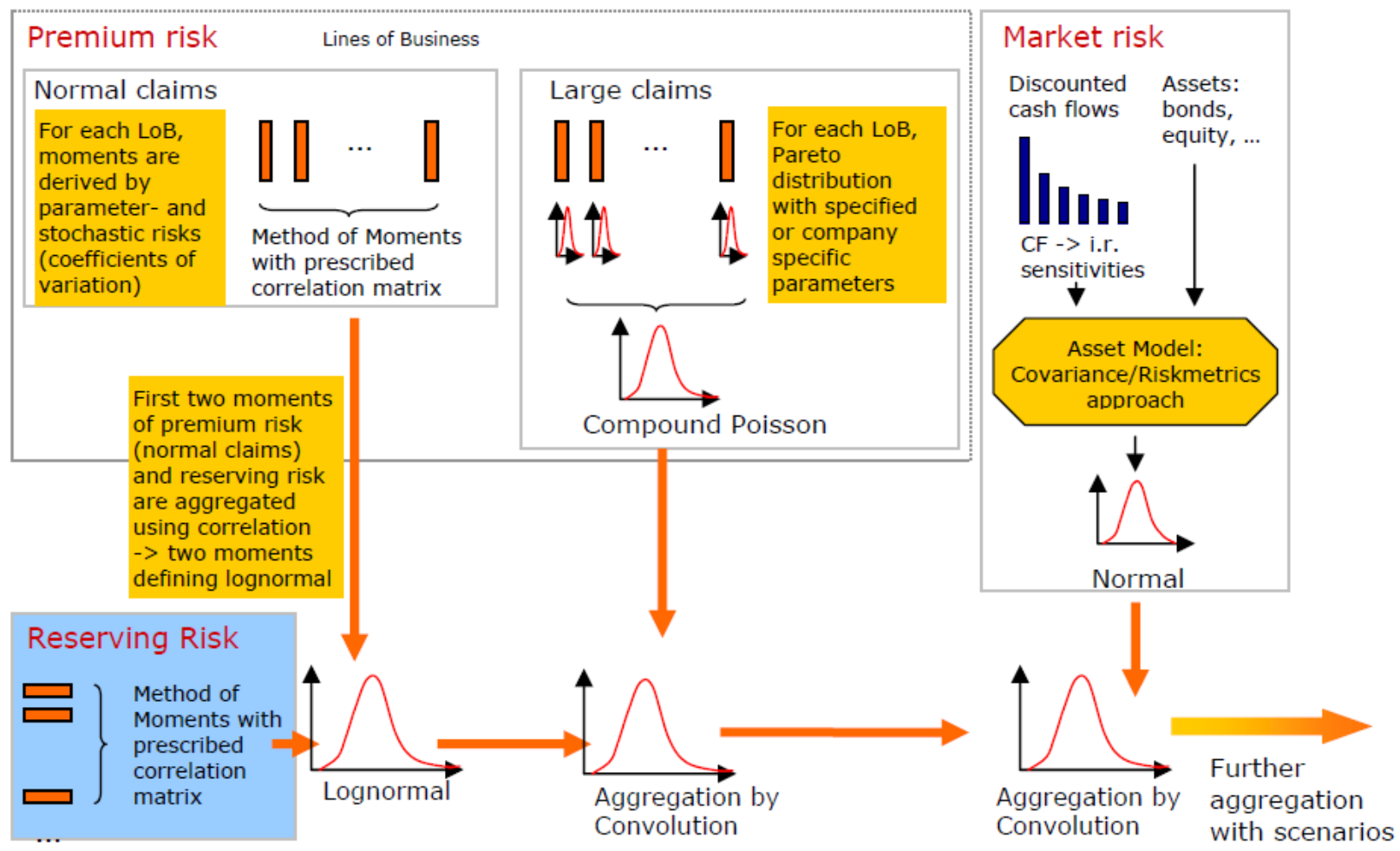

Source: White paper of the Swiss Solvency Test (Swiss Federal Office of Private Insurance, 2004) 


\section{Korean (In-sample estimation): between Jan 2002 and Dec 2016}

- Asset: seven benchmark monthly indices

- Underwriting: monthly aggregate claims from five lines of business

Panel A: Asset portfolio

\begin{tabular}{|c|c|c|c|c|c|c|c|c|}
\hline & mean & sd & skewness & kurtosis & Max & median & $\min$ & JB-test \\
\hline KR_stock (MSCl) & 0.0081 & 0.0771 & -0.3898 & 1.1737 & 0.2341 & 0.0087 & -0.3028 & $15.81^{\star \star \star}$ \\
\hline KR2Y & -0.0001 & 0.0118 & 0.0256 & 5.3170 & 0.0610 & 0.0019 & -0.0444 & $219.55^{\text {ᄎ** }}$ \\
\hline KR5Y & 0.0001 & 0.0173 & -0.5500 & 2.1224 & 0.0569 & 0.0030 & -0.0615 & $44.87^{\star \star \star \star ~}$ \\
\hline KR10Y & -0.0002 & 0.0252 & -0.4863 & 2.8502 & 0.1039 & 0.0038 & -0.0835 & $70.98^{\star \star \star *}$ \\
\hline KRcor & -0.0066 & 0.0528 & 0.2558 & 1.1983 & 0.1970 & -0.0098 & -0.1474 & $13.63^{\star \star \star}$ \\
\hline KR3MCD & -0.0065 & 0.0506 & -2.6660 & 13.0787 & 0.1130 & 0.0000 & -0.3270 & $1,535.30^{\star \star \star}$ \\
\hline Wrd_real (MSCI) & 0.0071 & 0.0564 & -1.3226 & 6.7998 & 0.2050 & 0.0129 & -0.3244 & $411.43^{\text {*ᄎ }}$ \\
\hline
\end{tabular}

Panel B: Insurance portfolio

(billion Korean Won)

\begin{tabular}{lrrrrrrr} 
& mean & sd & skewness & kurtosis & \multicolumn{1}{c}{ Max } & median & min \\
\hline Fire & 10.286 & 7.150 & 0.957 & 1.058 & 35.259 & 9.796 & 0.611 \\
Motor & $1,197.732$ & 713.675 & 0.464 & -0.437 & $3,288.883$ & $1,171.932$ & 117.852 \\
Marine & 45.707 & 31.177 & 0.494 & -0.793 & 123.084 & 39.943 & 1.827 \\
Liability & 24.113 & 16.376 & 0.886 & 0.399 & 74.868 & 21.304 & 1.548 \\
Accident & 82.782 & 69.597 & 0.823 & -0.460 & 272.329 & 58.581 & 1.578 \\
\hline
\end{tabular}


German (Out-of-sample estimation and Robustness check): between Jan 1998 and Dec 2006

- Asset: ten benchmark monthly indices

- Underwriting: monthly aggregate claims from six lines of business

Panel A: Asset portfolio

\begin{tabular}{lccrrrrrr} 
& Mean & sd & skewness & kurtosis & Max & median & \multicolumn{1}{c}{ min } & JB-test \\
\hline Wrd_stock (MSCl) & 0.005 & 0.041 & -0.679 & 0.673 & 0.089 & 0.009 & -0.140 & $11.007^{\star \star \star}$ \\
EMU_stock (MSCl) & 0.007 & 0.054 & -0.715 & 1.136 & 0.121 & 0.019 & -0.170 & $16.085^{\star \star \star}$ \\
DE_stock (MSCl) & 0.007 & 0.069 & -0.810 & 2.845 & 0.213 & 0.010 & -0.279 & $51.446^{\star \star \star}$ \\
US2Y & -0.001 & 0.100 & 0.149 & 2.337 & 0.378 & 0.003 & -0.301 & $27.139^{\star \star \star}$ \\
DE2Y & -0.001 & 0.069 & 0.078 & -0.319 & 0.177 & -0.002 & -0.136 & 0.436 \\
EMU2Y & 0.003 & 0.009 & -0.209 & -0.616 & 0.025 & 0.004 & -0.017 & 2.275 \\
IBOXX corp & 0.003 & 0.009 & -0.173 & -0.320 & 0.024 & 0.004 & -0.022 & 0.880 \\
EURIBOR3M & -0.000 & 0.044 & -0.452 & 2.279 & 0.135 & 0.001 & -0.151 & $29.245^{\star \star \star}$ \\
Wrd_real (MSCl) & 0.009 & 0.052 & 0.156 & 1.860 & 0.200 & 0.012 & -0.124 & $17.579^{\star \star \star}$ \\
Euro_real (MSCl) & 0.011 & 0.043 & -0.322 & 0.593 & 0.136 & 0.012 & -0.102 & 3.883 \\
\hline
\end{tabular}

Panel B: Insurance portfolio

\begin{tabular}{lrrrrrrr} 
& \multicolumn{1}{c}{ mean } & \multicolumn{1}{c}{ sd } & skewness & kurtosis & \multicolumn{1}{c}{ Max } & \multicolumn{1}{c}{ median } & min \\
\hline Ind_fire & $803,228.2$ & $1,240,581.2$ & 2.521 & 6.028 & $6,186,363.1$ & $295,276.2$ & 949.3 \\
HO_fire & $2,228,594.6$ & $991,556.9$ & -0.238 & 0.042 & $4,663,559.9$ & $2,251,845.3$ & $25,119.4$ \\
Other_fire & $1,313,010.5$ & $917,484.8$ & 1.054 & 0.543 & $4,146,761.6$ & $984,767.3$ & $3,384.7$ \\
HH_storm & $44,862.9$ & $74,288.1$ & 4.431 & 26.823 & $594,514.1$ & $18,223.3$ & 0.0 \\
HO_storm & $1,833,345.2$ & $3,990,165.4$ & 6.633 & 53.926 & $37,075,463.2$ & $462,420.4$ & $5,411.4$ \\
Water & $4,007,250.9$ & $1,445,809.7$ & -1.097 & 2.166 & $7,930,423.2$ & $4,277,080.0$ & $101,092.1$ \\
\hline
\end{tabular}

Risk aggregation in non-life insurance: Standard models vs. internal models 


\section{Marginal modeling: Asset portfolio}

- Korean data

\begin{tabular}{lcccc} 
& ARMA-order & AIC for ARMA & $\begin{array}{c}\text { Fitted distribution for } \\
\text { innovation }\end{array}$ & AIC for GARCH(1,1) \\
\hline KR_stock & $(0,3)$ & -416.37 & Skew normal & -439.28 \\
KR2Y & $(3,3)$ & $-1,119.34$ & Student-t & $-1,171.82$ \\
KR5Y & $(3,2)$ & -993.47 & Student-t & $-1,000.89$ \\
KR10Y & $(3,1)$ & -835.83 & Skew student & -841.92 \\
KRcor & $(0,1)$ & -552.81 & Student-t & -557.40 \\
KR3MCD & $(0,3)$ & -607.65 & Student-t & -696.40 \\
Wrd_real & $(2,3)$ & -540.72 & Skew normal & -591.04 \\
\hline
\end{tabular}

- German data

\begin{tabular}{|c|c|c|c|c|}
\hline & ARMA-order & AIC for ARMA & $\begin{array}{l}\text { Fitted distribution for } \\
\text { innovation }\end{array}$ & AIC for $\operatorname{GARCH}(1,1)$ \\
\hline Wrd_stock & $(0,0)$ & -379.11 & Skew normal & -396.16 \\
\hline EMU_stock & $(2,2)$ & -322.21 & Skew student & -351.18 \\
\hline DE_stock & $(0,0)$ & -269.68 & Skew student & -282.58 \\
\hline US2Y & $(0,0)$ & -188.98 & Student-t & -228.81 \\
\hline DE2Y & $(2,1)$ & -285.28 & Normal & -263.37 \\
\hline EMU2Y & $(1,1)$ & -735.19 & Skew normal & -706.72 \\
\hline IBOXX corp & $(3,0)$ & -729.12 & Normal & -720.41 \\
\hline EURIBOR3M & $(3,2)$ & -385.23 & Skew student & -400.88 \\
\hline Wrd_real & $(3,1)$ & -355.91 & Skew student & -346.53 \\
\hline Euro_real & $(0,1)$ & -380.42 & Skew normal & -367.99 \\
\hline
\end{tabular}

Risk aggregation in non-life insurance: Standard models vs. internal models 


\section{Marginal modeling: Underwriting portfolio}

- Korean data

\begin{tabular}{|c|c|c|c|c|c|}
\hline & Fire & Motor & Marine & Liability & Accident \\
\hline \multirow{2}{*}{ Skew Normal } & $6,347.08$ & $>10,000$ & $7,294.23$ & $6,657.01$ & $9,913.13$ \\
\hline & $\left(0.447^{\star \star \star}\right)$ & $(0.999 \star \star \star)$ & $\left(0.499^{\star \star \star}\right)$ & $\left(0.452^{\star \star \star}\right)$ & $\left(0.565^{\star \star \star}\right)$ \\
\hline \multirow{2}{*}{ Student-t } & $6,261.55$ & $8,138.76$ & $6,818.89$ & $6,716.72$ & $7,098.29$ \\
\hline & $\left(0.420^{\star \star \star}\right)$ & $\left(0.744^{\star \star \star}\right)$ & $\left(0.451^{\star \star \star}\right)$ & $\left(0.743^{\star \star \star}\right)$ & $\left(0.743^{\star \star \star}\right)$ \\
\hline \multirow{2}{*}{ Skew student-t } & $6,148.62$ & $7,845.05$ & $6,684.81$ & $6,452.62$ & $6,924.53$ \\
\hline & $(0.080)$ & $\left(0.113^{\star \star}\right)$ & $(0.059)$ & $(0.068)$ & $\left(0.115^{\star \star}\right)$ \\
\hline \multirow{2}{*}{ Lognormal } & $6,175.85$ & $7,860.66$ & $6,722.82$ & $6,466.80$ & $6,938.39$ \\
\hline & $\left(0.131^{\star \star \star}\right)$ & $\left(0.115^{\star \star}\right)$ & $\left(0.103^{\star \star}\right)$ & $(0.096 *)$ & $(0.084)$ \\
\hline \multirow{2}{*}{ Gamma } & $6,148.70$ & $7,834.37$ & $6,690.58$ & $6,445.75$ & $6,922.64$ \\
\hline & $(0.089)$ & $(0.081)$ & $(0.064)$ & $(0.045)$ & (0.065) \\
\hline \multirow{2}{*}{ Weibull } & $6,145.10$ & $7,826.92$ & $6,685.07$ & $6,443.84$ & $6,922.35$ \\
\hline & $(0.058)$ & $(0.051)$ & $(0.056)$ & $(0.034)$ & $(0.066)$ \\
\hline Inverse & $6,193.25$ & $>10,000$ & $6,747.59$ & $6,481.98$ & $>10,000$ \\
\hline Gaussian & $\left(0.185^{\star \star \star}\right)$ & $(0.999 \star \star \star)$ & $\left(0.172^{\star \star \star}\right)$ & $\left(0.148^{\star \star \star}\right)$ & $\left(0.999^{\star \star \star}\right)$ \\
\hline \multirow{2}{*}{ Cauchy } & $6,251.24$ & $7,934.52$ & $6,810.38$ & $6,548.92$ & $7,071.04$ \\
\hline & $(0.149 \star \star \star)$ & $\left(0.142^{\star \star \star}\right)$ & $\left(0.166^{\star \star \star}\right)$ & $\left(0.153^{\star \star \star}\right)$ & $\left(0.213^{\star \star \star}\right)$ \\
\hline \multirow{2}{*}{ Burr } & $7,057.71$ & $8,894.04$ & $7,619.40$ & $7,395.66$ & $7,799.34$ \\
\hline & $\left(0.570^{\star \star \star}\right)$ & $\left(0.593^{\star \star \star}\right)$ & $\left(0.566^{\star \star \star}\right)$ & $\left(0.574^{\star \star \star}\right)$ & $\left(0.565^{\star \star \star}\right)$ \\
\hline \multirow{2}{*}{ GPD } & $6,153.91$ & $7,838.88$ & $6,671.22$ & $6,454.96$ & $6,915.91$ \\
\hline & $(0.081)$ & $(0.080)$ & $(0.030)$ & $(0.066)$ & $\left(0.092^{*}\right)$ \\
\hline РОТ 90\% & $6,360.01$ & $9,625.44$ & $6,907.21$ & $6,666.27$ & $7,147.97$ \\
\hline (Norm-GPD) & $\left(0.445^{\star \star \star}\right)$ & $\left(0.825^{\star \star \star}\right)$ & $\left(0.438^{\star \star \star}\right)$ & $\left(0.447^{\star \star \star}\right)$ & $\left(0.438^{\star \star \star}\right)$ \\
\hline РОТ 90\% & $7,124.12$ & $8,962.02$ & $7,687.81$ & $7,466.74$ & $7,866.29$ \\
\hline (Lognorm-GPD) & $\left(0.786^{\star \star \star}\right)$ & $\left(0.804^{\star \star \star}\right)$ & $\left(0.728^{\star \star \star}\right)$ & $\left(0.725^{\star \star \star}\right)$ & $\left(0.735^{\star \star \star}\right)$ \\
\hline
\end{tabular}

\section{- German data}

\begin{tabular}{|c|c|c|c|c|c|c|}
\hline & Ind_fire & HO_fire & Otr_fire & HH_stm & HO_stm & Water \\
\hline Skew Normal & $\begin{array}{r}3,359.63 \\
\left(0.426^{\star \star \star}\right)\end{array}$ & $\begin{array}{r}3,452.52 \\
\left(0.549^{\star \star \star}\right)\end{array}$ & $\begin{array}{r}3,369.76 \\
\left(0.428^{\star \star \star}\right)\end{array}$ & $\begin{array}{r}2,732.08 \\
\left(0.274^{\star \star \star}\right)\end{array}$ & $\begin{array}{r}3,604.26 \\
\left(0.426^{\star \star \star}\right)\end{array}$ & $\begin{array}{r}3,572.18 \\
\left(0.600^{\star \star \star}\right)\end{array}$ \\
\hline Student & $\begin{array}{r}3,145.12 \\
\left(0.683^{\star * \star}\right)\end{array}$ & $\begin{array}{r}3,516.66 \\
\left(0.637^{\star \star *}\right)\end{array}$ & $\begin{array}{r}3,390.33 \\
\left(0.693^{\star * *}\right)\end{array}$ & $\begin{array}{r}2,795.55 \\
\left(0.487^{\star \star *}\right)\end{array}$ & $\begin{array}{r}3,295.83 \\
\left(0.655^{\star \star \star}\right)\end{array}$ & $\begin{array}{r}3,635.47 \\
\left(0.633^{\star \star \star}\right)\end{array}$ \\
\hline $\begin{array}{l}\text { Skew } \\
\text { student }\end{array}$ & $\begin{array}{r}3,128.23 \\
(0.056)\end{array}$ & $\begin{array}{r}3,337.36 \\
\left(0.309^{\star \star \star}\right)\end{array}$ & $\begin{array}{r}3,246.36 \\
(0.109)\end{array}$ & $\begin{array}{r}2,514.79 \\
(0.075)\end{array}$ & $\begin{array}{r}3,292.71 \\
(0.093)\end{array}$ & $\begin{array}{r}3,457.85 \\
\left(0.441^{\star \star \star}\right)\end{array}$ \\
\hline Lognormal & $\begin{array}{r}3,137.60 \\
(0.103)\end{array}$ & $\begin{array}{r}3,391.84 \\
\left(0.267^{\star \star \star}\right)\end{array}$ & $\begin{array}{l}3,285.51 \\
\left(0.148^{\star \star}\right)\end{array}$ & $\begin{array}{r}2,529.24 \\
(0.077)\end{array}$ & $\begin{array}{r}3,289.55 \\
(0.095)\end{array}$ & $\begin{array}{r}3,520.44 \\
\left(0.377^{\star \star \star}\right)\end{array}$ \\
\hline Gamma & $\begin{array}{l}3,140.02 \\
\left(0.133^{\star \star}\right)\end{array}$ & $\begin{array}{r}3,337.01 \\
\left(0.210^{\star \star *}\right)\end{array}$ & $\begin{array}{r}3,247.31 \\
(0.087)\end{array}$ & $\begin{array}{r}2,514.24 \\
(0.079)\end{array}$ & $\begin{array}{r}3,310.62 \\
\left(0.164^{\star \star \star}\right)\end{array}$ & $\begin{array}{r}3,455.35 \\
\left(0.345^{\star \star \star}\right)\end{array}$ \\
\hline Weibull & $\begin{array}{r}3,134.27 \\
(0.100)\end{array}$ & $\begin{array}{l}3,311.14 \\
\left(0.141^{\star \star}\right)\end{array}$ & $\begin{array}{r}3,244.18 \\
(0.068)\end{array}$ & $\begin{array}{r}2,509.65 \\
(0.073)\end{array}$ & $\begin{array}{l}3,300.03 \\
\left(0.149^{\star *}\right)\end{array}$ & $\begin{array}{r}3,409.59 \\
\left(0.282^{\star \star \star}\right)\end{array}$ \\
\hline $\begin{array}{l}\text { Inverse } \\
\text { Gaussian }\end{array}$ & $\begin{array}{r}3,256.48 \\
\left(0.411^{\star \star \star}\right)\end{array}$ & $\begin{array}{r}3,462.41 \\
\left(0.428^{\star \star \star}\right)\end{array}$ & $\begin{array}{r}3,418.60 \\
\left(0.421^{\star \star *}\right)\end{array}$ & $\begin{array}{r}2,958.41 \\
\left(0.776^{\star \star \star}\right)\end{array}$ & $\begin{array}{r}3,337.78 \\
\left(0.213^{\star \star \star}\right)\end{array}$ & $\begin{array}{r}3,582.77 \\
\left(0.506^{\star \star \star}\right)\end{array}$ \\
\hline Cauchy & $\begin{array}{r}3,203.37 \\
\left(0.220^{\star * \star}\right)\end{array}$ & $\begin{array}{r}3,321.02 \\
(0.078)\end{array}$ & $\begin{array}{l}3,289.30 \\
\left(0.139^{\star \star}\right)\end{array}$ & $\begin{array}{r}2,606.33 \\
\left(0.252^{\star \star \star}\right)\end{array}$ & $\begin{array}{r}3,381.99 \\
\left(0.264^{\star \star *}\right)\end{array}$ & $\begin{array}{r}3,309.34 \\
(0.062)\end{array}$ \\
\hline Burr & $\begin{array}{r}3,499.17 \\
\left(0.509^{\star \star *}\right)\end{array}$ & $\begin{array}{r}3,907.38 \\
\left(0.540^{\star \star *}\right)\end{array}$ & $\begin{array}{r}3,759.83 \\
\left(0.540^{\star * *}\right)\end{array}$ & $\begin{array}{r}2,813.60 \\
\left(0.501^{\star * *}\right)\end{array}$ & $\begin{array}{r}3,669.61 \\
\left(0.529^{\star * *}\right)\end{array}$ & $\begin{array}{r}4,048.19 \\
\left(0.536^{\star \star *}\right)\end{array}$ \\
\hline GPD & $\begin{array}{r}3,128.36 \\
(0.070)\end{array}$ & $\begin{array}{r}3,315.54 \\
\left(0.240^{\star \star \star}\right)\end{array}$ & $\begin{array}{r}3,247.90 \\
(0.114)\end{array}$ & $\begin{array}{r}2,509.01 \\
(0.061)\end{array}$ & $\begin{array}{r}3,289.94 \\
(0.114)\end{array}$ & $\begin{array}{r}3,430.96 \\
\left(0.369^{\star \star \star}\right)\end{array}$ \\
\hline Norm-GPD & $\begin{array}{r}3,267.40 \\
\left(0.215^{\star \star \star}\right)\end{array}$ & $\begin{array}{r}3,296.22 \\
(0.070)\end{array}$ & $\begin{array}{r}3,266.07 \\
\left(0.160^{\star \star \star}\right)\end{array}$ & $\begin{array}{r}2,639.78 \\
\left(0.201^{\star \star \star}\right)\end{array}$ & $\begin{array}{r}3,451.95 \\
\left(0.220^{\star \star \star}\right)\end{array}$ & $\begin{array}{r}3,371.74 \\
\left(0.246^{\star \star \star}\right)\end{array}$ \\
\hline LN-GPD & $\begin{array}{r}3,513.02 \\
\left(0.657^{\star \star \star}\right)\end{array}$ & $\begin{array}{r}3,403.94 \\
\left(0.389^{\star \star \star}\right)\end{array}$ & $\begin{array}{r}3,797.46 \\
\left(0.741^{\star \star \star}\right)\end{array}$ & $\begin{array}{r}2,552.05 \\
\left(0.207^{\star \star \star}\right)\end{array}$ & $\begin{array}{r}3,709.15 \\
\left(0.694^{\star \star \star}\right)\end{array}$ & $\begin{array}{r}4,085.97 \\
\left(0.742^{\star \star *}\right)\end{array}$ \\
\hline
\end{tabular}

Risk aggregation in non-life insurance: Standard models vs. internal models Martin Eling and Kwangmin Jung ARIA 2018 


\section{Decision Criteria and Goodness-of-fit (Korean data)}

- Base-level

\begin{tabular}{|c|c|c|c|c|c|c|c|}
\hline \multirow{2}{*}{ Family } & \multirow[b]{2}{*}{ Copula } & \multicolumn{3}{|c|}{ Asset Portfolio } & \multicolumn{3}{|c|}{ Insurance Portfolio } \\
\hline & & Log-lik & $\mathrm{AIC}$ & GoF & Log-lik & AIC & GoF \\
\hline \multirow{2}{*}{ Elliptical } & Gaussian & 164.04 & -286.07 & $0.018^{\star \star}$ & 619.23 & $-1,218.45$ & $0.144^{\star \star *}$ \\
\hline & Student-t & 191.07 & -338.14 & 0.012 & 620.75 & $-1,219.49$ & $0.114^{\star \star \star}$ \\
\hline \multirow{2}{*}{ Archimedean } & Gumbel & 0.183 & 1.634 & $0.041^{* * *}$ & 344.48 & -686.96 & $0.810^{\star * *}$ \\
\hline & Clayton & 3.617 & -5.234 & $0.025^{*}$ & 418.30 & -834.59 & $2.735^{\star \star \star}$ \\
\hline \multirow{2}{*}{ HAC } & Gumbel & 109.28 & -206.56 & $0.082^{*}$ & 474.65 & -941.30 & $0.039 * *$ \\
\hline & Clayton & 99.43 & -186.87 & $0.082^{*}$ & 488.22 & -968.44 & $0.039 * *$ \\
\hline PCC & R-Vine & 210.81 & -377.62 & 161.03 & 660.53 & $-1,303.06$ & 75.44 \\
\hline \multicolumn{2}{|c|}{ Bernstein (D-Vine) } & 207.70 & -60.47 & - & 638.96 & -504.67 & - \\
\hline
\end{tabular}

- Top-level

\begin{tabular}{|c|c|c|c|}
\hline & & Statistics & P-value \\
\hline Independence test & & 0.8586 & 0.3906 \\
\hline \multirow{2}{*}{ Elliptical } & Gaussian copula & $0.3860 * \star$ & 0.0400 \\
\hline & Student-t copula & $0.3893^{\star \star}$ & 0.0300 \\
\hline \multirow{2}{*}{ Archimedean } & Gumbel copula & $0.3907^{\star \star}$ & 0.0200 \\
\hline & Clayton copula & $0.3952^{\star \star \star}$ & 0.0000 \\
\hline
\end{tabular}




\section{Decision Criteria and Goodness-of-fit (German data)}

- Base-level

\begin{tabular}{|c|c|c|c|c|c|c|c|}
\hline \multirow{2}{*}{ Family } & \multirow[b]{2}{*}{ Copula } & \multicolumn{3}{|c|}{ Asset Portfolio } & \multicolumn{3}{|c|}{ Insurance Portfolio } \\
\hline & & Log-lik & $\mathrm{AIC}$ & GoF & Log-lik & AIC & GoF \\
\hline \multirow{2}{*}{ Elliptical } & Gaussian & 269.35 & -448.70 & $0.033^{\star *}$ & 175.74 & -321.47 & $0.119 * * *$ \\
\hline & Student-t & 285.41 & -478.82 & 0.024 & 175.75 & -319.49 & $0.100^{\star \star \star}$ \\
\hline \multirow{2}{*}{ Archimedean } & Gumbel & 48.86 & -95.72 & $0.036^{\star *}$ & 76.87 & -151.73 & $0.230 * \star \star$ \\
\hline & Clayton & 68.03 & -134.07 & $0.022^{*}$ & 122.78 & -243.56 & 0.055 \\
\hline \multirow{2}{*}{ HAC } & Gumbel & 243.39 & -468.78 & 0.175 & 142.77 & -275.54 & 0.642 \\
\hline & Clayton & 208.74 & -339.48 & 0.875 & 162.36 & -314.73 & 0.759 \\
\hline PCC & R-Vine & 311.27 & -580.53 & 71.36 & 203.00 & -374.00 & 56.43 \\
\hline \multicolumn{2}{|c|}{ Bernstein (D-Vine) } & 310.30 & -32.34 & - & 181.88 & -73.72 & - \\
\hline
\end{tabular}

- Top-level

\begin{tabular}{llcc} 
& & Statistics & P-value \\
\hline Independence test & & 0.7593 & 0.4477 \\
\hline \multirow{2}{*}{ Elliptical } & Gaussian copula & 0.2334 & 0.1300 \\
& Student-t copula & 0.2782 & 0.1500 \\
\hline \multirow{2}{*}{ Archimedean } & Gumbel copula & $0.2356^{\star}$ & 0.0900 \\
& Clayton copula & $0.2714^{\star \star}$ & 0.0400 \\
\hline
\end{tabular}




\section{Capital requirement (Korean data)}

Top-level dependence (Bivariate)

\begin{tabular}{|c|c|c|c|c|c|c|c|}
\hline \multirow{2}{*}{\multicolumn{3}{|c|}{ (in trillion) }} & \multirow{2}{*}{\multicolumn{2}{|c|}{ Indep }} & \multirow{2}{*}{\multicolumn{3}{|c|}{ Gumbel }} \\
\hline & & & & & & & \\
\hline \multirow{21}{*}{ 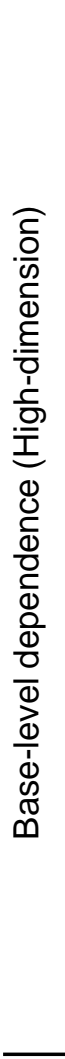 } & \multirow{7}{*}{ VaR at $99 \%$} & R-Vine & 25.33 & 25.61 & 25.62 & 25.54 & 25.50 \\
\hline & & Gauss & 25.19 & 25.40 & 25.43 & 25.37 & 25.33 \\
\hline & & $\mathrm{t}$ & 25.59 & 25.82 & 25.83 & 25.78 & 25.72 \\
\hline & & HAC-best & 24.41 & 24.73 & 24.75 & 24.64 & 24.61 \\
\hline & & HAC-worst & 27.03 & 27.27 & 27.27 & 27.22 & 27.18 \\
\hline & & Independence & 20.71 & 20.97 & 21.01 & 20.91 & 20.88 \\
\hline & & Bernstein & 22.10 & 22.19 & 22.14 & 22.18 & 22.12 \\
\hline & \multirow{7}{*}{ VaR at $99.5 \%$} & R-Vine & 28.87 & 29.21 & 29.33 & 29.45 & 29.05 \\
\hline & & Gauss & 28.62 & 28.93 & 29.03 & 29.07 & 28.83 \\
\hline & & $\mathrm{t}$ & 29.38 & 29.67 & 29.80 & 29.84 & 29.58 \\
\hline & & HAC-best & 27.22 & 27.38 & 27.50 & 27.54 & 27.32 \\
\hline & & HAC-worst & 30.91 & 31.25 & 31.38 & 31.46 & 31.10 \\
\hline & & Independence & 23.63 & 23.77 & 23.85 & 24.01 & 23.70 \\
\hline & & Bernstein & 25.07 & 25.24 & 25.29 & 25.27 & 25.17 \\
\hline & \multirow{7}{*}{ TVaR at $99 \%$} & R-Vine & 29.32 & 29.60 & 29.64 & 29.72 & 29.55 \\
\hline & & Gauss & 28.78 & 29.06 & 29.10 & 29.13 & 28.98 \\
\hline & & $\mathrm{t}$ & 29.67 & 29.97 & 30.00 & 30.07 & 29.89 \\
\hline & & HAC-best & 27.73 & 27.97 & 27.97 & 28.03 & 27.91 \\
\hline & & HAC-worst & 31.08 & 31.37 & 31.42 & 31.48 & 31.30 \\
\hline & & Independence & 24.07 & 24.33 & 24.32 & 24.39 & 24.29 \\
\hline & & Bernstein & 25.24 & 25.52 & 25.56 & 25.53 & 25.39 \\
\hline & & & \multicolumn{2}{|c|}{ K-RBC (VaR 99\%) } & SII (VaR 99.5\%) & \multicolumn{2}{|c|}{ SST (TVaR 99\%) } \\
\hline \multicolumn{3}{|c|}{ Regulations (in trillion) } & 39.07 & & 38.12 & \multicolumn{2}{|c|}{35.34} \\
\hline
\end{tabular}

Risk aggregation in non-life insurance: Standard models vs. internal models Martin Eling and Kwangmin Jung 


\section{Capital requirement (German data)}

Top-level dependence (Bivariate)

\begin{tabular}{|c|c|c|c|c|c|c|c|}
\hline \multirow{2}{*}{\multicolumn{3}{|c|}{ (in € million) }} & & & & & \\
\hline & & & Indep & Gauss & $\mathrm{t}$ & Gumbel & Clayton \\
\hline \multirow{21}{*}{ 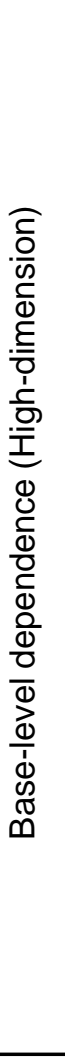 } & \multirow{7}{*}{ VaR at $99 \%$} & R-Vine & 242.72 & 259.60 & 267.97 & 270.56 & 267.97 \\
\hline & & Gauss & 218.98 & 237.95 & 247.69 & 250.03 & 247.69 \\
\hline & & $\mathrm{t}$ & 224.38 & 240.81 & 248.52 & 250.68 & 248.52 \\
\hline & & HAC-best & 152.89 & 168.96 & 179.42 & 182.46 & 179.42 \\
\hline & & HAC-worst & 283.33 & 299.01 & 306.44 & 309.16 & 306.44 \\
\hline & & Independence & 46.41 & 69.68 & 79.05 & 81.33 & 67.79 \\
\hline & & Bernstein & 297.03 & 301.44 & 305.89 & 307.05 & 299.10 \\
\hline & \multirow{7}{*}{ VaR at $99.5 \%$} & R-Vine & 411.32 & 415.71 & 416.93 & 422.23 & 413.48 \\
\hline & & Gauss & 388.33 & 392.05 & 392.95 & 399.76 & 389.09 \\
\hline & & $\mathrm{t}$ & 401.40 & 407.06 & 407.57 & 414.53 & 404.65 \\
\hline & & HAC-best & 307.97 & 311.76 & 312.00 & 315.89 & 309.58 \\
\hline & & HAC-worst & 477.37 & 480.44 & 484.26 & 494.37 & 476.84 \\
\hline & & Independence & 203.66 & 207.25 & 207.70 & 212.18 & 205.13 \\
\hline & & Bernstein & 355.50 & 363.49 & 363.11 & 365.52 & 363.39 \\
\hline & \multirow{7}{*}{ TVaR at $99 \%$} & R-Vine & 417.22 & 420.57 & 423.64 & 428.45 & 417.48 \\
\hline & & Gauss & 397.17 & 401.04 & 404.58 & 409.43 & 398.66 \\
\hline & & $\mathrm{t}$ & 412.50 & 416.45 & 420.26 & 425.55 & 413.56 \\
\hline & & HAC-best & 310.63 & 314.21 & 317.43 & 321.15 & 311.99 \\
\hline & & HAC-worst & 486.15 & 490.43 & 494.24 & 500.88 & 487.66 \\
\hline & & Independence & 204.38 & 207.38 & 210.24 & 213.99 & 205.80 \\
\hline & & Bernstein & 374.92 & 374.74 & 375.98 & 379.79 & 371.96 \\
\hline & & & \multicolumn{2}{|c|}{ K-RBC (VaR 99\%) } & \multicolumn{2}{|c|}{ SII (VaR 99.5\%) } & SST (TVaR 99\%) \\
\hline \multicolumn{3}{|c|}{ Regulations (in € million) } & \multicolumn{2}{|c|}{613.63} & \multicolumn{2}{|c|}{600.35} & 561.67 \\
\hline
\end{tabular}

Risk aggregation in non-life insurance: Standard models vs. internal models Martin Eling and Kwangmin Jung ARIA 2018 


\section{Findings and Implications}

- R-Vine copula model with flexible choice on dependence functions is superior to any other high-dimensional models for both Korean and German datasets.

- $35 \%$ overestimation on average among three regulatory frameworks takes place.

- In the size of the overestimation, 18 percentage points out of 35 result from the correlation assumption and 17 points from the calibrated risk parameters.

- Specifically, Korean RBC generates the highest gap between the estimated model and the standard model among three regulations, which we conclude might come from no diversification in the market risk module and less categorization in the underwriting module.

- The size of the overestimation can be larger for small- and mid-sized company.

\section{Limitations and Future research}

- Life underwriting module

- Other risk modules in SII, e.g., operational risk 


\section{Thank you for your attention!}




\section{Capital requirement: asset allocation}

Panel A: Asset allocations

\begin{tabular}{llrr} 
& & Allocation 1 & Allocation 2 \\
\hline Equity & KR_stock & $10 \%$ & $30 \%$ \\
Fixed income & KR2Y & $20 \%$ & $10 \%$ \\
& KR5Y & $20 \%$ & $10 \%$ \\
& KR10Y & $20 \%$ & $10 \%$ \\
Money Market & KRcor & $15 \%$ & $10 \%$ \\
Real estate & KR3MCD & $0 \%$ & $0 \%$ \\
\hline
\end{tabular}

Real estate Wrd_real

(in trillion)

Top-level dependence

\begin{tabular}{|c|c|c|c|c|c|c|c|c|}
\hline & & & Indep & Gauss & $\mathrm{t}$ & Gumbel & Clayton & K-RBC \\
\hline \multirow{15}{*}{ 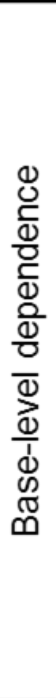 } & \multirow{7}{*}{$\begin{array}{l}\text { Allocation } 1 \\
\text { (VaR 99\%) } \\
\text { - } \quad \mu=4.09 \% \\
\text { - } \quad \sigma=25.06 \% \\
\text { - } \quad \mathrm{RP}=0.46 \%\end{array}$} & PCC & 26.42 & 26.69 & 26.88 & 26.74 & 26.46 & \multirow{7}{*}{36.82} \\
\hline & & Gauss & 26.45 & 26.71 & 26.85 & 26.73 & 26.50 & \\
\hline & & $t$ & 26.66 & 26.95 & 27.12 & 26.95 & 26.70 & \\
\hline & & HAC-best & 25.34 & 25.61 & 25.81 & 25.71 & 25.39 & \\
\hline & & HAC-worst & 27.90 & 28.16 & 28.31 & 28.16 & 27.93 & \\
\hline & & Independence & 21.91 & 22.15 & 22.36 & 22.24 & 21.95 & \\
\hline & & Bernstein & 22.45 & 22.60 & 22.59 & 22.48 & 22.46 & \\
\hline & & & Indep & Gauss & $\mathrm{t}$ & Gumbel & Clayton & K-RBC \\
\hline & \multirow{7}{*}{$\begin{array}{l}\text { Allocation } 2 \\
\text { (VaR 99\%) } \\
\text { - } \quad \mu=5.04 \% \\
\text { - } \quad \sigma=28.02 \% \\
\text { - } \quad \mathrm{RP}=0.60 \%\end{array}$} & PCC & 27.53 & 27.74 & 27.75 & 27.74 & 27.60 & \multirow{7}{*}{42.98} \\
\hline & & Gauss & 27.32 & 27.47 & 27.51 & 27.53 & 27.39 & \\
\hline & & $\mathrm{t}$ & 27.61 & 27.80 & 27.82 & 27.85 & 27.70 & \\
\hline & & HAC-best & 26.22 & 26.45 & 26.48 & 26.45 & 26.28 & \\
\hline & & HAC-worst & 28.61 & 28.78 & 28.80 & 28.83 & 28.68 & \\
\hline & & Independence & 23.31 & 23.54 & 23.50 & 23.48 & 23.36 & \\
\hline & & Bernstein & 26.23 & 26.23 & 26.25 & 26.25 & 26.24 & \\
\hline
\end{tabular}

Risk aggregation in non-life insurance: Standard models vs. internal models Martin Eling and Kwangmin Jung ARIA 2018 


\section{Diversification Effect (Korean data) for the asset portfolio}

$$
d i v=\frac{\operatorname{SCR}\left(\sum_{i=1}^{d} X_{i}\right)-\sum_{i=1}^{d} S C R_{i}}{\sum_{i=1}^{d} S C R_{i}}
$$

where $\operatorname{SCR}\left(\sum_{i=1}^{d} X_{i}\right)$ is the solvency capital requirement of a portfolio with d-dimensional risks and $\sum_{i=1}^{d} S C R_{i}$ is the sum of individual SCRs for the portfolio.

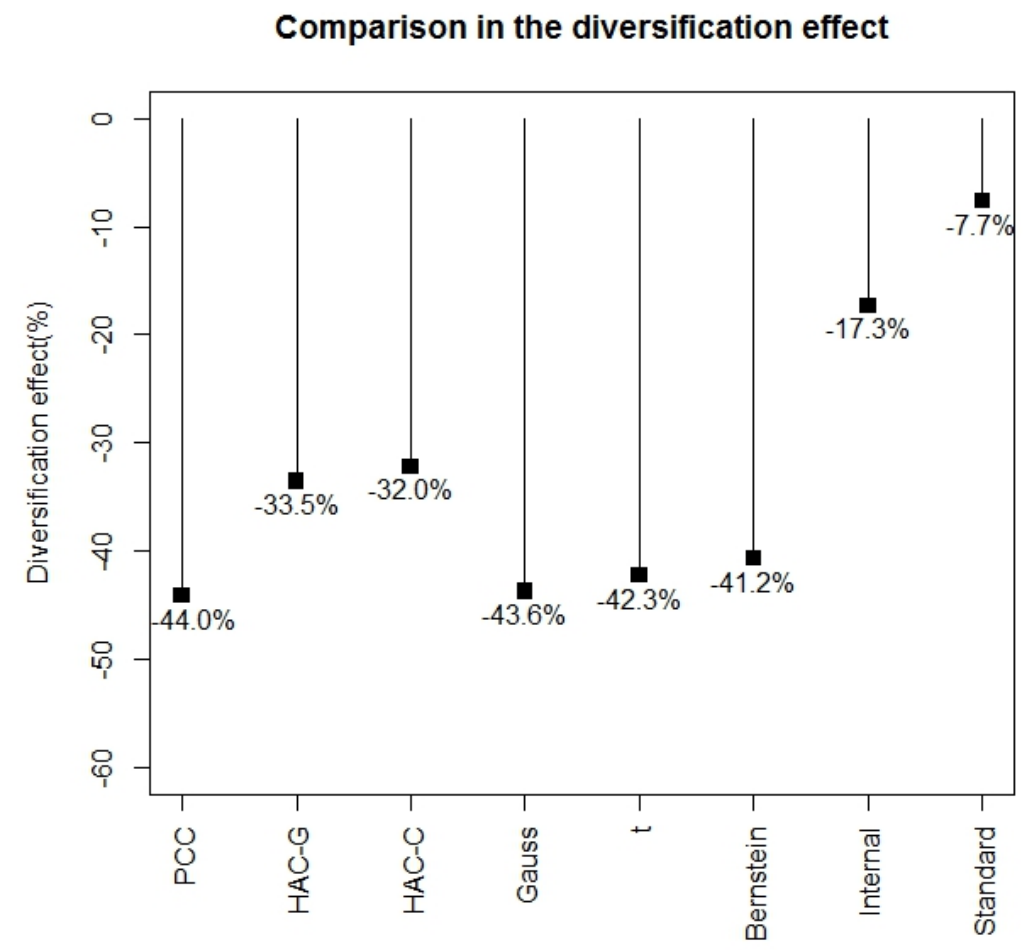

- "Internal" indicates the diversification benefit from the internal model in Gatzert and Martin (2012) and "Standard" from the standard model in the same paper. 